\title{
Stability of Barley stripe mosaic virus-Induced Gene Silencing in Barley
}

\author{
Marianne Bruun-Rasmussen, Christian Toft Madsen, Stine Jessing, and Merete Albrechtsen \\ Department of Genetics and Biotechnology, Faculty of Agricultural Sciences, University of Aarhus, Thorvaldsensvej 40, Opg. \\ 8, 2. sal, 1871 Frederiksberg C, Denmark.
}

Submitted 2 May 2007. Accepted 24 June 2007.

Virus-induced gene silencing (VIGS) can be used as a powerful tool for functional genomics studies in plants. With this approach, it is possible to target most genes and downregulate the messenger $(\mathrm{m}) \mathrm{RNA}$ in a sequence-specific manner. Barley stripe mosaic virus (BSMV) is an established VIGS vector for barley and wheat; however, silencing using this vector is generally transient, with efficient silencing often being confined to the first two or three systemically infected leaves. To investigate this further, part of the barley Phytoene desaturase (PDS) gene was inserted into BSMV and the resulting photobleaching in infected barley plants was used as a reporter for silencing. In addition, downregulation of $P D S$ mRNA was measured by quantitative reverse-transcriptase polymerase chain reaction (qRTPCR). Using fragments of $P D S$ ranging from 128 to 584 nucleotides in BSMV, we observed that insert length influenced stability but not efficiency of VIGS. Silencing was transient in most cases; however, the decrease in PDS mRNA levels measured by qRT-PCR began earlier and lasted longer than the photobleaching. Occasionally, silencing persisted and could be transmitted through seed as well as via mechanical inoculation, although large parts of the insert had been lost from the virus vector. The instability of the insert, observed consistently throughout our experiments, offers an explanation for the transient nature of silencing when using BSMV as a VIGS vector.

Additional keywords: Hordeum vulgare, insert stability, monocots, time course, VIGS efficiency.

Virus-induced gene silencing (VIGS) has been established as a tool for functional genomics in a range of dicot plant species, including Nicotiana spp. (tobacco), Pisum sativum (pea), Arabidopsis, Solanum lycopersicum (tomato), and several others (Constantin et al. 2004; Dalmay et al. 2000; Kumagai et al. 1995; Liu et al. 2002; Ruiz et al. 1998). Also, the monocot species Hordeum vulgare (barley), Triticum aestivum (wheat), Oryza sativum (rice), and Zea mays (maize) have been targeted

M. Bruun-Rasmussen and C. T. Madsen contributed equally to this work. Corresponding author: M. Albrechtsen; E-mail: m.albrechtsen@dias.kvl.dk

Current address of Stine Jessing: BioCentrum, Technical University of Denmark, Matematiktorvet, Building 301, 2. sal, 2800 Kgs. Lyngby, Denmark.

* The $\boldsymbol{e}$-Xtra logo stands for "electronic extra" and indicates Figure 2 is published in color online. by VIGS (Ding et al. 2006; Holzberg et al. 2002; Scofield et al. 2005). The use of VIGS for functional genomics exploits the fact that plant viruses trigger a plant defense mechanism, related to post-transcriptional gene silencing, where doublestranded RNA is degraded into short interfering RNAs (siRNAs). A gene of interest can be introduced into a virus vector and the recombinant virus will trigger silencing of both the virus carrying the insert and the homologous endogenous plant messenger (m)RNAs (Brodersen and Voinnet 2006; Burch-Smith et al. 2004). Silencing initiated by VIGS will spread systemically along with the virus (Schwach et al. 2005; Voinnet et al. 2000) and, by this method, it is possible to downregulate almost any gene of interest if a suitable vector is present for the plant species under investigation.

Monocots are laborious to transform (Altpeter et al. 1996); instead, a biolistic approach to express or silence genes transiently has been used for functional genomics in barley and wheat. However, this method is limited to a local reaction and the bombardment procedure leads to tissue damage that may influence the results (Schweizer et al. 1999a,b, 2000). As an alternative, a VIGS vector based on Barley stripe mosaic virus (BSMV) was used for silencing of genes associated with powdery mildew resistance in barley and leaf rust resistance or tan spot disease in wheat (Hein et al. 2005; Scofield et al. 2005; Shen et al. 2007; Tai et al. 2007). The tripartite positive-sense RNA virus BSMV is one of only two VIGS vectors currently reported suitable for silencing in monocot species, including barley and wheat (Holzberg et al. 2002; Scofield et al. 2005; Tai et al. 2005), the other being Brome mosaic virus (Ding et al. 2006). The three BSMV components are designated $\alpha, \beta$, and $\gamma$ and have a methylated 5' cap and a $3^{\prime}$ polyadenylate sequence followed by a tyrosine accepting structure (Agranovsky et al. 1979, 1982). The $\alpha$ component of the BSMV ND18 strain is $3.8 \mathrm{~kb}$ and encodes an RNA-dependent RNA polymerase (RdRP) (Petty et al. 1990). The $\beta$ component is $3.2 \mathrm{~kb}$ and directly encodes the capsid protein $(\mathrm{CP})$ and, indirectly, via two subgenomic RNAs, four proteins $\left(\beta b, \beta c, \beta d\right.$, and $\left.\beta d^{\prime}\right)$ necessary for systemic movement of the virus. These four proteins are collectively referred to as the triple gene block (TGB) proteins (Gustafson and Armour 1986; Petty and Jackson 1990; Zhou and Jackson 1996). The smallest RNA component, designated $\gamma$, is $2.8 \mathrm{~kb}$ and encodes an additional subunit of the RdRP and the multifunctional $\gamma \mathrm{b}$ protein (Fig. 1A) (Bragg and Jackson 2004; Petty et al. 1990). Silencing using BSMV as a VIGS vector has been accomplished by inserting part of a gene of interest either closely after the stop codon of the $\gamma b$ gene (Holzberg et al. 2002) or at the beginning of the $\gamma \mathrm{b}$ gene, preventing the expression of the $\gamma \mathrm{b}$ (Tai et al. 2005). 
Photobleaching caused by silencing of Phytoene desaturase $(P D S)$ is often used as the reporter in VIGS studies because it produces an obvious phenotype (Brigneti et al. 2004; Constantin et al. 2004; Kumagai et al. 1995; Ratcliff et al. 2001; Ruiz et al. 1998). Variation in PDS silencing has been observed when using BSMV as a VIGS vector, with the most extensive silencing occurring in the fifth leaf at 10 to 17 days after inoculation of the second leaf (Holzberg et al. 2002). Although several groups have explored the potential of BSMV as a VIGS vector, a detailed investigation of the factors influencing the stability of silencing in barley has not been performed. One reason for the transient silencing observed in this and other VIGS systems could be a partial or complete loss of the insert via recombination during virus replication. The size of the insert may influence the stability of recombinant viruses. In recombinant Potato virus $X$ (PVX), insert stability was shown to be inversely correlated to size during multiple passages in Nicotiana benthamiana (Avesani et al. 2007). Recent studies suggest that insert stability also may be affected by the nature of the insert. In a green fluorescent protein (GFP) transgenic $N$. benthamiana plant, recombinant Tomato bushy stunt virus maintained a GFP insert better than two inserts of endogene plant origin (Zhong et al. 2005). Finally, temperature may also influence insert stability (Qu et al. 2005).

In this report, we investigate some factors affecting the stability of gene silencing in barley using BSMV as a VIGS vector, and we present a detailed account of the parameters influencing

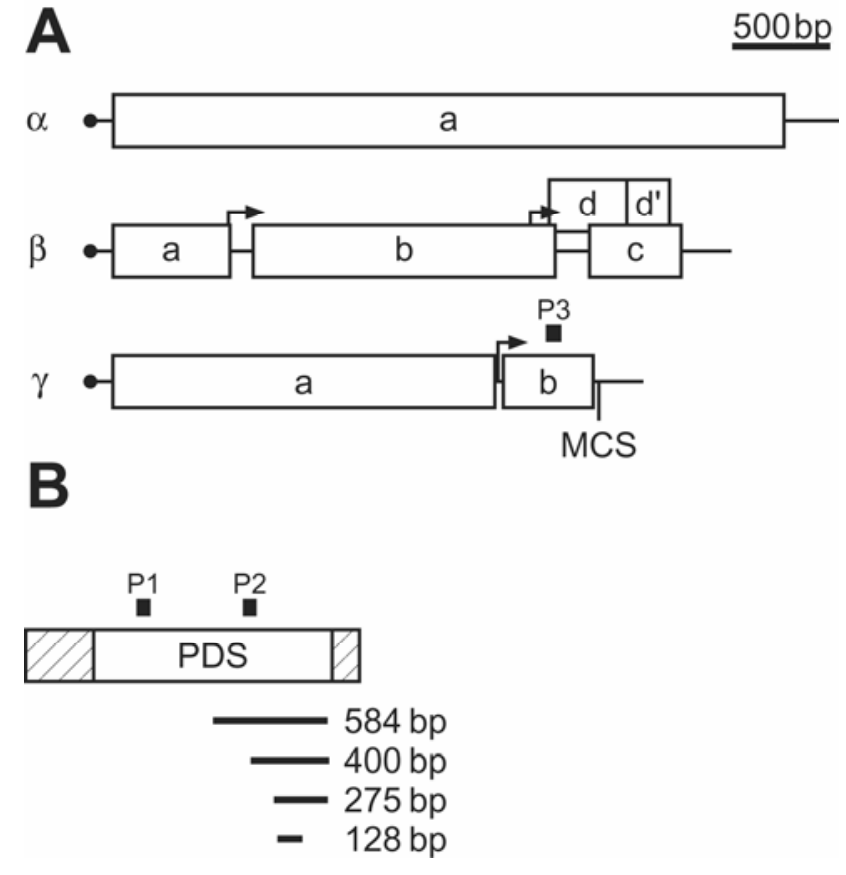

Fig. 1. Schematic organization of the Barley stripe mosaic virus (BSMV) genome and the Phytoene desaturase (PDS) inserts. A, Genomic organization of the three BSMV components $\alpha, \beta$, and $\gamma$ drawn to scale. Open reading frames are indicated by boxes and the $5^{\prime}$ end of subgenomic RNAs by arrows. The multiple cloning site (MCS) is positioned downstream of the $\gamma \mathrm{b}$ gene. The amplicon from the primer/probe set (P3) used for quantification of BSMV by quantitative reverse-transcriptase polymerase chain reaction (qRT-PCR) is indicated. $\mathbf{B}$, Schematic representation of the PDS open reading frame drawn to the same scale as above. The hatched box represents the $P D S$ full-length open reading frame from Oryza sativum (rice), and the open box within represents the available corresponding partial sequence of $P D S$ from barley. The position and size of the fragments used for virus-induced gene silencing (VIGS) are shown below and the amplicons corresponding to two qRT-PCR primer/probe sets P1 and P2 are indicated. The amplicon from P2 partially overlaps the 400 nucleotides of $P D S$ used for construction of BSMV $\mathrm{PDS}_{\mathrm{P} 00}$. virus-induced gene silencing in this virus-host system. In order to investigate silencing efficiency and insert stability, we have tested different insert lengths, time points, and virus passages in several generations. We show here that VIGS efficiency was largely unaffected by insert size and orientation between 584 and 128 nucleotides (nt). However, the propensity to maintain the insert was negatively correlated with size, which significantly influenced virus abundance. When measuring silencing over time with quantitative reverse-transcriptase polymerase chain reaction (qRT-PCR), a reduction in mRNA level was present before and lasted longer than the visual silencing. Surprisingly, part of the insert was lost even before photobleaching was visible. Even though silencing with BSMV was transient, photobleaching occasionally persisted and was observed after seed transmission and mechanical inoculation, although the virus had lost a substantial portion of the insert. Once the insert is below approximately $100 \mathrm{nt}$, it appears to be stable. The transient nature of silencing using BSMV as a VIGS vector can be explained by the insert instability presented here.

\section{RESULTS}

Determining optimal conditions

for VIGS in barley using BSMV as a VIGS vector.

A multiple cloning site was engineered into the $\gamma$ component of BSMV downstream of the $\gamma \mathrm{b}$ gene, following the strategy of Holzberg and associates (2002). Subsequently, $400 \mathrm{nt}$ of PDS from barley were inserted into the multiple cloning site $\left(\mathrm{BSMV}_{\mathrm{PDS} 400}\right)$ (Fig. 1). The BSMV $\mathrm{PDS}_{400}$ was used for determining the optimal conditions for silencing in barley. First, conditions for measuring PDS mRNA by qRT-PCR were established by carrying out an experiment to determine whether the qRT-PCR results were influenced by the position of the primer/probe set. Two primer/probe sets were tested: one corresponding to a region $358 \mathrm{nt}$ upstream from the part of the $P D S$ gene inserted in the virus vector $(\mathrm{P} 1)$ and the other set corresponding to a region partially overlapping the part of $P D S$ used for BSMV $\mathrm{PDS}_{400}$ (P2) (Fig. 1B). The first leaf on barley (H. vulgare) cv. Black Hulless plants was mechanically inoculated with RNA transcribed from clones of the $\alpha, \beta$, and $\gamma$ components of BSMV. By 14 days postinoculation (dpi), the lamina of the third leaf was harvested, RNA was isolated, and the relative amounts of PDS mRNA were assessed by qRTPCR ( $\Delta \Delta$ CT method) (Livak and Schmittgen 2001) using the two Taqman primer/probe sets targeting PDS. No significant differences in the $P D S$ mRNA quantities were determined with the two primer/probe sets (data not shown). The primer/probe set P1 was chosen for further experiments. We tested two genes for normalization: 18S rRNA and glyceraldehyde-3-phosphate dehydrogenase (GAPDH). No significant differences between them were detected. Because the primer/probe set for $18 \mathrm{~S}$ rRNA gave the highest PCR efficiency, we chose this primer/probe set for further experiments (data not shown).

Previously, improved photobleaching was observed with BSMV having a deletion in the $\beta a$ gene $(\beta \Delta \beta a)$, suggesting that the use of $\beta \Delta \beta$ a could improve BSMV as a VIGS vector (Holzberg et al. 2002). This variant BSMV construct subsequently has been used for silencing of genes involved in the resistance of barley to powdery mildew (Hein et al. 2005). With an equivalent $\beta \Delta \beta$ a construct, we observed that any enhanced photobleaching in barley was masked by extensive necrosis (data not shown). We conclude that $\beta \Delta \beta \mathrm{a}$, in our hands, is unsuitable for VIGS studies in barley; a similar conclusion was reached by Scofield and associates (2005).

In order to determine an optimal cultivar for VIGS studies, seven different barley cultivars (Black Hulless, Golden Promise, Pallas, Ingrid, Chess, Simba, and Relief) were tested with 
$\mathrm{BSMV}_{\mathrm{PDS} 400}$ (data not shown). Although some degree of photobleaching was observed in all cultivars tested, maximum photobleaching was obtained in Black Hulless which, therefore, was chosen for further experiments. To determine to what extent temperature affects BSMV-induced $P D S$ silencing, we set up a VIGS experiment at $16,20,24$, and $28^{\circ} \mathrm{C}$. Ten Black Hulless barley plants inoculated with $\mathrm{BSMV}_{\mathrm{PDS} 400}$ were placed in each growth chamber, and the plants were assessed for the extent of photobleaching and virus symptoms until 14 dpi. No significant differences in photobleaching were observed between the 20 and $24^{\circ} \mathrm{C}$ groups. The plants at $16^{\circ} \mathrm{C}$ exhibited a delayed onset and reduced extent of photobleaching. The plants at $28^{\circ} \mathrm{C}$ exhibited a slower growth and also very limited silencing (data not shown). Taken together, our studies show that the conditions for optimal silencing with BSMV as a VIGS vector are obtained without the $\beta \Delta \beta$ a deletion in cv. Black Hulless at 20 or $24^{\circ} \mathrm{C}$. The quantification of $P D S$ mRNA by qRT-PCR was best performed using the P1 primer/probe set and using 18S rRNA for normalization.

\section{Insert length influences stability but not efficiency of VIGS.}

Different lengths of $P D S$ from barley inserted into the multiple cloning site of BSMV were tested in order to investigate to what extent the insert size affects VIGS efficiency and stability. In addition to $\mathrm{BSMV}_{\mathrm{PDS} 400}$, we constructed $\mathrm{BSMV}_{\mathrm{PDS} 584}$, $\mathrm{BSMV}_{\mathrm{PDS} 275}$, and $\mathrm{BSMV}_{\mathrm{PDS} 128}$ carrying 584, 275, and $128 \mathrm{nt}$, respectively, of $P D S$ (Fig. 1B). Six days after sowing, groups of 10 plants were inoculated with RNA from either wild-type virus or one of the four recombinant viruses. One group was left uninoculated. The lamina of the third leaf from the first five plants showing virus symptoms in each group was harvested at $14 \mathrm{dpi}$ and the level of photobleaching was assessed. Unambiguous photobleaching was observed in all plants inoculated with $\mathrm{BSMV}_{\mathrm{PDS} 584}, \mathrm{BSMV}_{\mathrm{PDS} 400}$, and $\mathrm{BSMV}_{\mathrm{PDS} 275}$. However, the area affected by photobleaching was smaller in plants inoculated with $\mathrm{BSMV}_{\mathrm{PDS} 584}$ than in those inoculated with $\mathrm{BSMV}_{\mathrm{PDS} 400}$ and $\mathrm{BSMV}_{\mathrm{PDS} 275}$. Plants infected with $\mathrm{BSMV}_{\mathrm{PDS} 128}$ only rarely showed the completely white areas typical for PDS silencing; instead, the leaves developed very pale green or pale yellow stripes. These symptoms were clearly distinct from virus symptoms. The onset of this phenotype was observed later than the white photobleached areas seen in the other groups. A representative leaf from each of the groups inoculated with $\mathrm{BSMV}_{\mathrm{WT}}, \mathrm{BSMV}_{\mathrm{PDS} 400}$, or $\mathrm{BSMV}_{\mathrm{PDS} 128}$ is shown in Figure 2A. Although typical photobleaching was not seen in plants inoculated with $\mathrm{BSMV}_{\mathrm{PDS} 128}$, qRT-PCR revealed an $84 \%$ downregulation of $P D S$ mRNA relative to plants inoculated with BSMV $\mathrm{WT}_{\mathrm{WT}}$ (Fig. 2A and B). Similar downregulation of PDS mRNA in plants inoculated with $\mathrm{BSMV}_{\mathrm{PDS} 128}$ was observed consistently in several experiments (data not shown). Plants inoculated with $\mathrm{BSMV}_{\mathrm{PDS} 584}, \mathrm{BSMV}_{\mathrm{PDS} 400}$, and BSMV $_{\text {PDS275 }}$ showed around 68 to $78 \%$ downregulation of PDS mRNA (Fig. 2B). Overall, the visual photobleaching did not correlate closely with the amount of PDS mRNA measured by qRT-PCR. Uninoculated plants had a lower level of PDS mRNA than plants inoculated with BSMV ${ }_{\mathrm{wT}}$ (Fig. 2B). Induction of PDS mRNA following BSMV inoculation has been observed in several independent experiments.

Insert stability in relation to the insert length was investigated by PCR using primers flanking the insert. The cDNA from the qRT-PCR analysis was used as template for the PCR (Fig. 2D). The shortest PDS insert (128 nt) was still intact after the 2 -week duration of the experiment. $\mathrm{BSMV}_{\mathrm{PDS} 584}$ with the longest insert was the most unstable, having lost most of the insert by $14 \mathrm{dpi}$. The two intermediate-size constructs, BSMV $\mathrm{PDS}_{275}$ and $\mathrm{BSMV}_{\mathrm{PDS} 400}$, still carried most of the insert by $14 \mathrm{dpi}$, although PCR species representing partial deletions also were obtained.
The relative virus accumulation was measured by qRT-PCR using the same cDNA as used for measuring the PDS mRNA level (Fig. 2C). The primer/probe (P3) set used for BSMV measurements amplifies a small part of the $\gamma b$ gene upstream of the inserts (Fig. 1A). Except for plants inoculated with BSMV ${ }_{\text {PDS584, }}$ where most of the insert was lost, virus accumulation correlated negatively with the insert length. The mean virus abundance in plants inoculated with BSMV $\mathrm{PDS584}_{\text {in }}$ was not significantly higher than that of plants inoculated with BSMV $\mathrm{WT}_{\mathrm{WT}}$.

To test whether the orientation of the insert was important for VIGS efficiency, we tested two additional constructs: $\mathrm{BSMV}_{\mathrm{PDS} 400 \mathrm{AS}}$ and $\mathrm{BSMV}_{\mathrm{PDS} 128 \mathrm{AS}}$, carrying the 400- or 128-nt $P D S$ fragments, respectively, in antisense orientation. We did not detect any visual differences in the photobleaching or any differences in efficiency measured by qRT-PCR between sense and antisense constructs (data not shown).

In previous studies with other RNA viruses, the majority of viral-derived siRNAs were found to originate from highly basepaired regions of the viral plus strand (Molnar et al. 2005). Furthermore, modifications of stem-loop hairpins to remove bulges and produce more perfectly basepaired dsRNA structures have enabled up to three times more efficient Dicer cleavage in vitro (Landry and Perreault 2005). Computer predictions by the Mfold program (Zuker et al. 1999) suggested that the 128-nt $P D S$ fragment used for $\mathrm{BSMV}_{\mathrm{PDS} 128}$ forms two stem-loop hairpins with only a few mismatches or bulges (data not shown); therefore, $13 \mathrm{nt}$ were changed by site-directed mutagenesis to remove these predicted bulges and form two perfectly basepaired predicted hairpins. However, no further improvement in silencing efficiency was obtained (data not shown). For convenience, the subsequent work was carried out only with unmutagenized sense constructions.

\section{The transient nature of $P D S$ silencing over time correlates with insert instability.}

Throughout our experiments, BSMV-induced PDS silencing often was found to be transient. However, in a few cases, photobleaching persisted throughout the life of the plant and even reappeared in progeny plants. To investigate the transient nature of BSMV silencing further, silencing with BSMV $\mathrm{PDS}_{400}$ was followed during a time period of 4 weeks. As controls, we inoculated barley with $\mathrm{BSMV}_{\mathrm{WT}}$. The laminas from two leaves from each of six plants inoculated with either $\mathrm{BSMV}_{\mathrm{PDS} 400}$ or $\mathrm{BSMV}_{\mathrm{WT}}$ were collected at 5, 7, 9, and 14 dpi and 4 weeks postinoculation (wpi). The photobleaching and the virus symptoms on the leaves were visually scored in $25 \%$ increments (Fig. 3). BSMV symptoms appeared $5 \mathrm{dpi}$ and increased until 75 to $100 \%$ of the leaf surfaces displayed symptoms in the last part of the experiment (Fig. 3D). Photobleaching appeared by $7 \mathrm{dpi}$ and had almost disappeared by $4 \mathrm{wpi}$, with an optimum of photobleaching in the third leaf around 14 dpi (Fig. 3B). Reductions in PDS mRNA levels between 48 and $92 \%$ relative to $\mathrm{BSMV}_{\mathrm{WT}}$ were observed during the time course experiment (Fig. 3A). A significant decrease of $65 \%$ of the relative amount of PDS mRNA was observed even before photobleaching appeared (student $t$ test, 5\% confidence level). The maximal silencing was found in the third leaf at $7 \mathrm{dpi}$ and the fourth leaf at 9 dpi and, in both cases, the $P D S$ mRNA level was reduced by $92 \%$. Surprisingly, a $48 \%$ reduction in $P D S$ mRNA was seen after photobleaching had almost completely disappeared by 4 wpi. When we repeated the time course experiment, a 10 to $75 \%$ reduction in PDS mRNA was observed. A substantial variation in $P D S$ expression was observed between the two experiments. However, the degree of silencing, calculated as the reduction in the $P D S$ mRNA levels in the $\mathrm{BSMV}_{\mathrm{PDS}}$ group relative to the $\mathrm{BSMV}_{\mathrm{WT}}$ group, was reproducible between the two experiments. In the repetition experiment, the photobleaching 
A

BSMV $_{\text {WT }}$

BSMV $_{\text {PDS400 }}$

$\mathrm{BSMV}_{\mathrm{PDS} 128}$

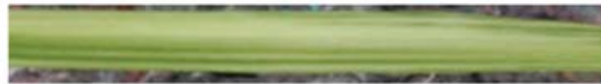

B
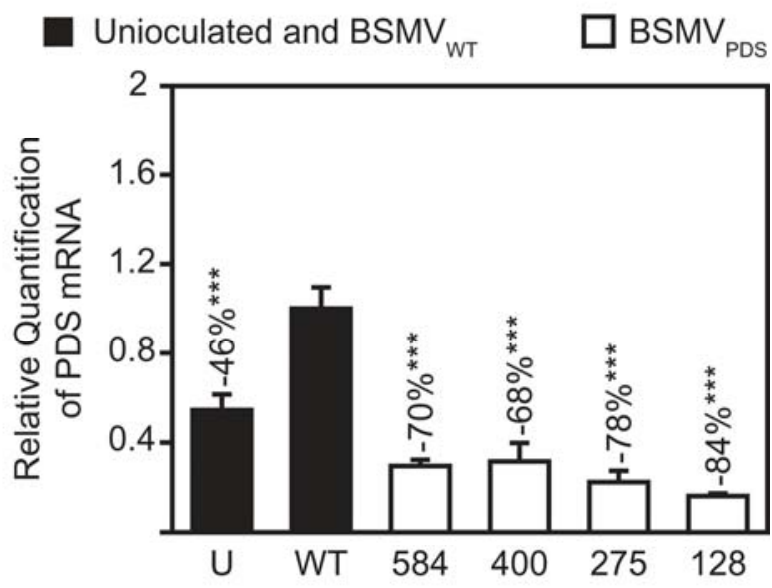

C
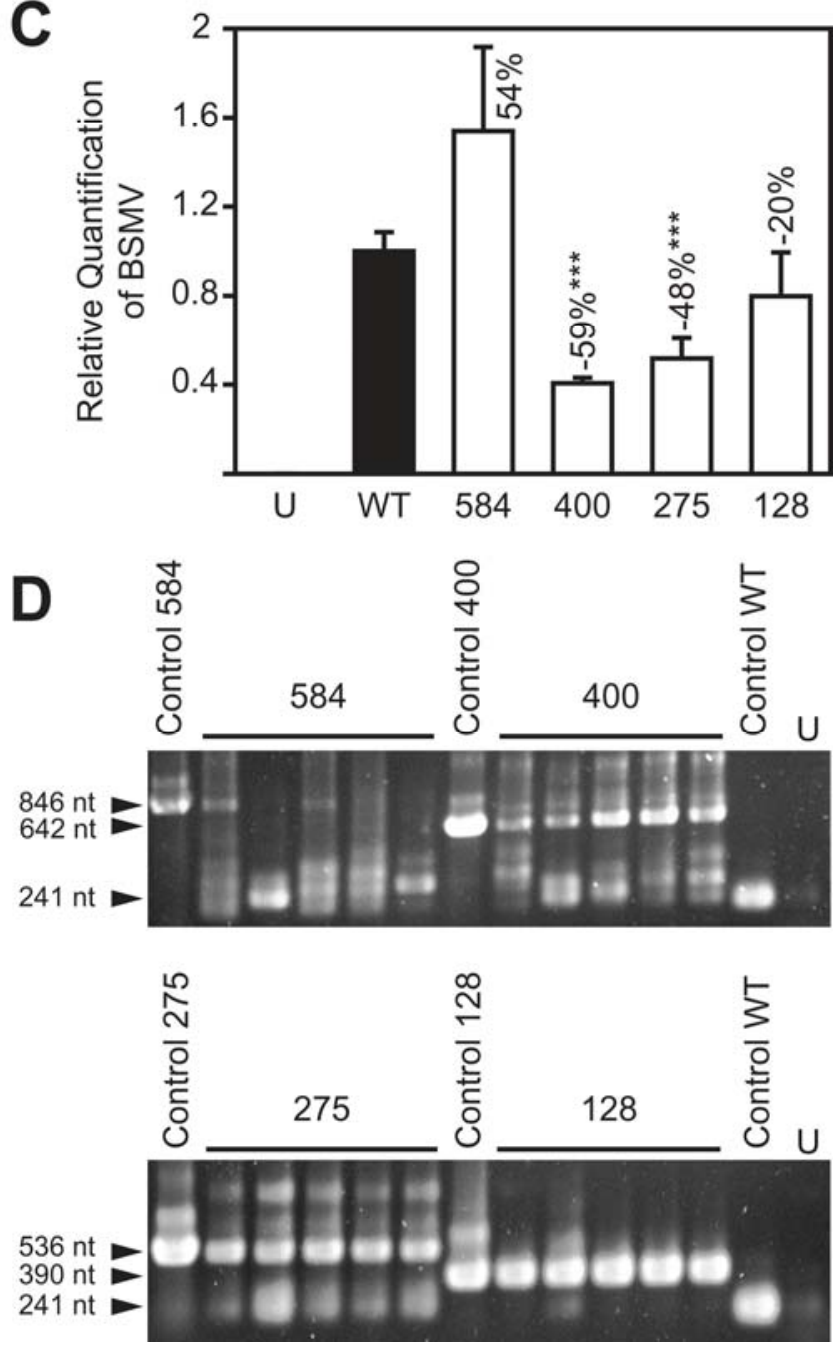

had disappeared completely by 4 wpi; however, a $50 \%$ reduction of the PDS mRNA level was still observed at this time point (data not shown). Silencing of $P D S$ did not correlate with BSMV accumulation measured by qRT-PCR using the P3 primer/probe set previously described. During the experiment, BSMV accumulation tended to increase in agreement with the increase in virus symptoms (Fig. 3C and D).

The stability of the $P D S$ insert over time was analyzed by PCR on the cDNA from the time course experiments. Already by $7 \mathrm{dpi}$, partial loss of the insert was observed (Fig. 3E). At 9 dpi, partial loss of the insert was seen in all the plants and, by $14 \mathrm{dpi}$, only a small part of the original insert was still present. The insert seemed to be completely lost by 4 wpi but, nevertheless, silencing was still present (Fig. 3A and E).

\section{VIGS is seed and mechanically transmittable.}

The seed transmission of BSMV can be as high as $61 \%$ depending on the virus strain and the barley cultivar tested (Edwards 1995; Timian 1974). For that reason, we decided to test the possibility of seed transmission of VIGS using BSMV. Ten plants were inoculated with $\mathrm{BSMV}_{\mathrm{PDS} 400}$ and subsequently observed during the entire period of growth and maturation. The most extensive photobleaching was seen in the third leaf, with leaves developing later showing less silencing. In some plants, all visual signs of $P D S$ silencing had disappeared before the onset of flowering; however, in 4 of the 10 plants, some degree of photobleaching was still seen in the flag leaves and the aristas of at least some of the heads. A total of 182 seeds from these four plants germinated. The development in symptoms and photobleaching were followed during the first month after sowing. In this experiment, 14\% (26 of 182) of the second-generation plants displayed virus symptoms and 5\% (10 of 182) developed signs of photobleaching. To our knowledge, this is the first report of seed transmission of silencing using VIGS. Of 65 seeds harvested from plants where silencing had disappeared before flowering, 10 second-generation plants developed virus symptoms, but none exhibited $P D S$ silencing. In order to investigate how much of the original $P D S$ insert was retained in plants grown from infected seed, RNA was isolated 19 days after sowing from five second-generation randomly chosen plants showing photobleaching and seven displaying only BSMV symp-

Fig. 2. Phytoene desaturase $(P D S)$ silencing, virus accumulation, and insert stability in plants inoculated with Barley stripe mosaic virus (BSMV) carrying different lengths of PDS. Groups of 10 plants were left uninoculated (U) or inoculated with $\mathrm{BSMV}_{\mathrm{WT}}(\mathrm{WT}), \mathrm{BSMV}_{\mathrm{PDS} 584}$ (584), $\mathrm{BSMV}_{\mathrm{PDS} 400}$ (400), BSMV ${ }_{\mathrm{PDS} 275}$ (275), or BSMV $\mathrm{PDS} 128$ (128). A, Photos at 14 days postinoculation (dpi) of representative barley leaves infected with $\mathrm{BSMV}_{\mathrm{WT}}, \mathrm{BSMV}_{\mathrm{PDS} 400}$, or $\mathrm{BSMV}_{\mathrm{PDS} 128}$. B, Relative quantification (RQ) of $P D S$ mRNA performed by quantitative reverse-transcriptase polymerase chain reaction (qRT-PCR) with the P1 primer/probe-set normalized to $18 \mathrm{~S}$ rRNA using the $\Delta \Delta \mathrm{CT}$ method (Livak and Schmittgen 2001). Averages from five plants from each group are shown. The average RQ of plants inoculated with $B S M V_{W T}$ is set to be 1 . The changes in RQ relative to the $\mathrm{BSMV}_{\mathrm{WT}}$ group are indicated as percentages. Error bars represent the standard error of the mean. Asterisks ( $* * *)$ denote $0.1 \%$ level of confidence (student $t$ test) for the difference between the RQ values of each group (U, 584, 400, 275, and 128) compared with the group inoculated with BSMV $\mathrm{WT}_{\mathrm{WT}}(\mathrm{WT})$. C, Average RQ of the BSMV $\gamma \mathrm{b}$ gene as assessed by qRT-PCR with the P3 primer/probe-set normalized to $18 \mathrm{~S}$ rRNA. The average RQ of plants inoculated with BSMV $\mathrm{WT}_{\mathrm{WT}}$ is set to be 1 . The changes in $R Q$ compared with the $\mathrm{BSMV}_{\mathrm{WT}}$ group are indicated as percentages. $\mathbf{D}$, cDNA prepared for qRT-PCR (B and C) was used for PCR amplification using primers flanking the insert. As size markers, PCR products amplified from plasmid $\gamma$ components were used. The size of the amplicon is $241 \mathrm{nu}-$ cleotides (nt) for pBSMV $\mathrm{WT}_{\mathrm{WT}}$ (Control WT), $846 \mathrm{nt}$ for $\mathrm{pBSMV}_{\mathrm{PDS} 584}$ (Control 584), $642 \mathrm{nt}$ for $\mathrm{pBSMV}_{\mathrm{PDS} 400}\left(\right.$ Control 400), $536 \mathrm{nt}$ for $\mathrm{pBSMV}_{\mathrm{PDS} 275}$

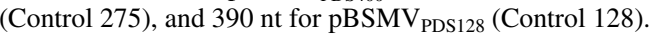


toms. RT-PCR was carried out as described above with primers flanking the insert and the products were analyzed by sequencing (Fig. 4A). In all plants tested, the virus had partly or completely lost the PDS insert, with less extensive deletions generally being seen in samples from plants showing photobleaching than from those showing only virus symptoms (Fig. 4B).
In a separate experiment, seed were harvested from a plant displaying long-lasting photobleaching after inoculation with $\mathrm{BSMV}_{\mathrm{PDS} 400}$. Five second-generation plants displayed virus symptoms and three of these also showed photobleaching. RTPCR and sequencing of RNA isolated from these plants revealed the same 315-nt deletion of the original insert (Fig. 4C). New

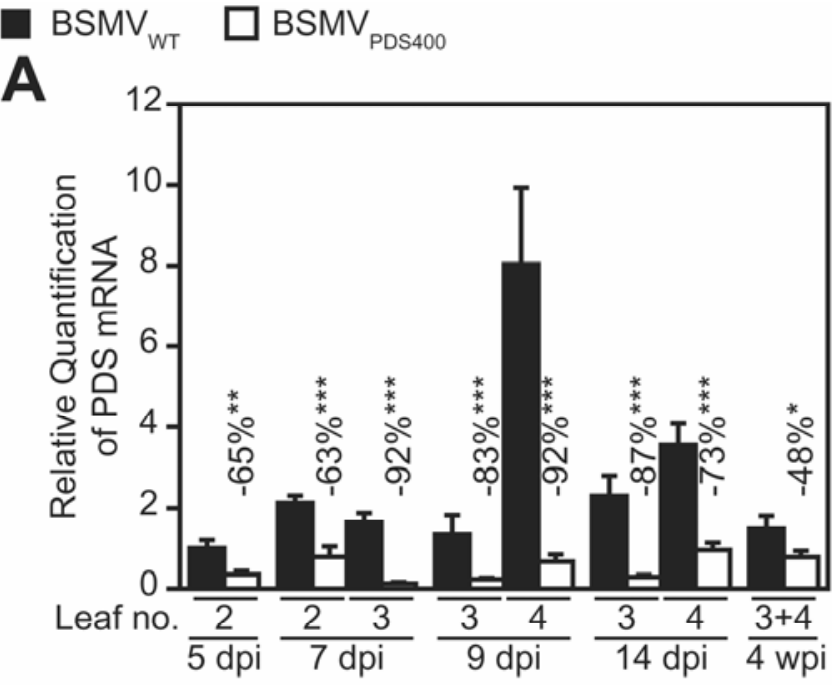

B
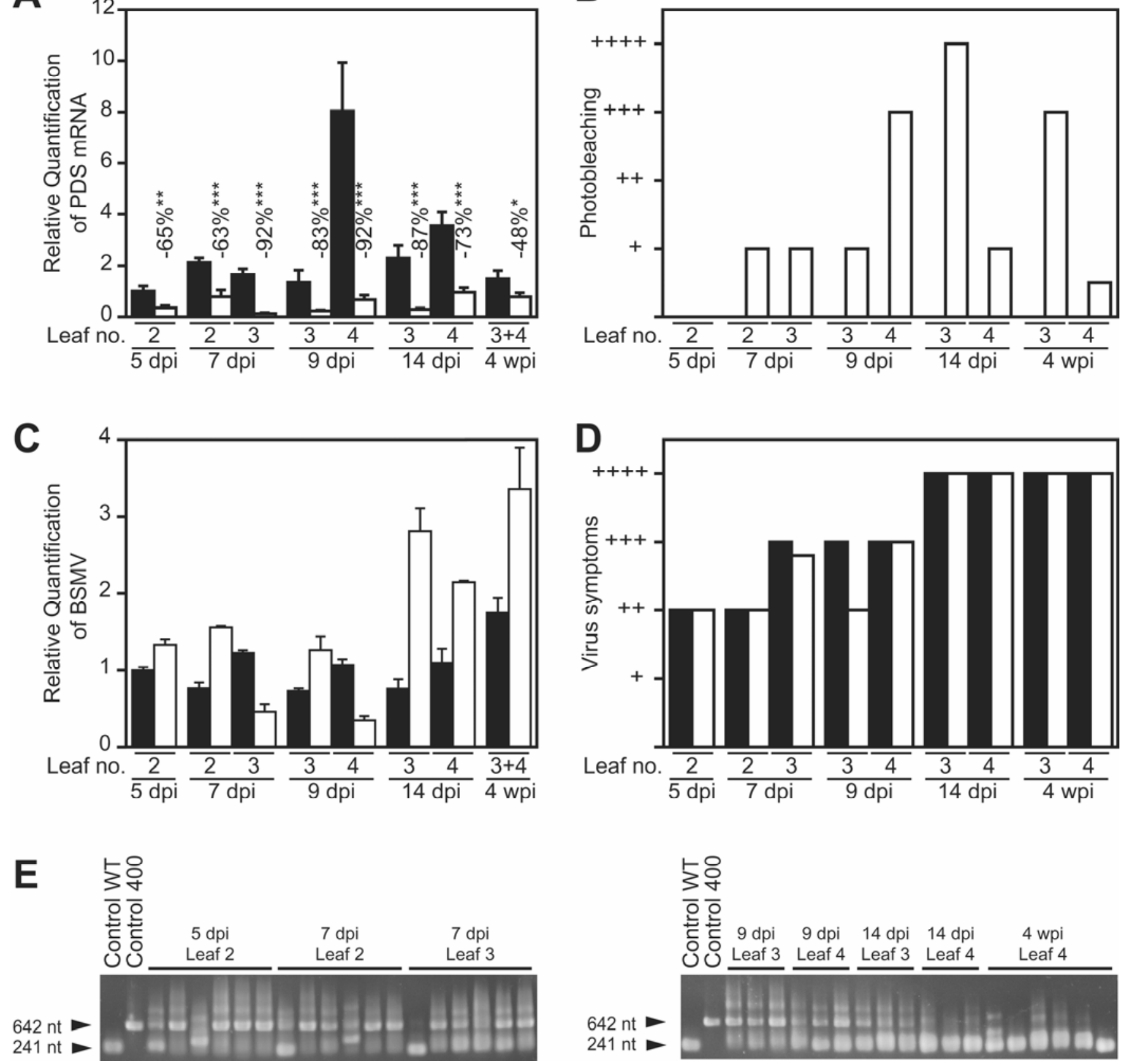

Fig. 3. Phytoene desaturase (PDS) silencing, virus accumulation, and insert stability over time. Groups of six plants were inoculated with Barley stripe mosaic virus wild type $\left(\mathrm{BSMV}_{\mathrm{WT}}\right)$ (black bars) or $\mathrm{BSMV}_{\mathrm{PDS} 400}$ (white bars) and harvested at different time points up to 4 weeks postinoculation. $\mathbf{A}$, Relative quantification of PDS mRNA. The changes in PDS mRNA levels of BSMV $\mathrm{PDS} 400_{\text {infected leaves compared with the BSMV }} \mathrm{WT}_{\mathrm{T}}$ control groups are shown as percentages. Error bars represent the standard error of the mean. Asterisks denote the level of confidence for the difference between the plants inoculated with $\mathrm{BSMV}_{\mathrm{WT}}$ and the plants inoculated with $\mathrm{BSMV}_{\mathrm{PDS} 400}$ at the same time point (student $t$ test; ***, **, and $*=0.1,5$, and $1 \%$, respectively). B, Visual assessment of photobleaching by $25 \%$ increments in each leaf. The visual evaluation is the average assessment of six individual plants. C, Average relative quantification of the BSMV $\gamma \mathrm{b}$ gene measured by quantitative reverse-transcriptase polymerase chain reaction (qRT-PCR) with the P3 primer/probe-set normalized to $18 \mathrm{~S}$ rRNA. Error bars represent the standard error of the mean. D, Visual assessment of virus symptoms in $25 \%$ increments. The visual evaluation is the average assessment of six individual plants. E, cDNA prepared for qRT-PCR (A and C) was used for PCR amplification using primers flanking the insert. As size markers, PCR products amplified from plasmid $\gamma$ components were used. The size of the amplicon is 241 nucleotides (nt) for

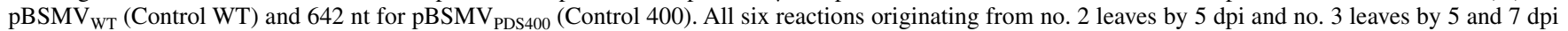
are shown in the left panel, whereas only three representative reactions from leaves harvested at later time points are shown in the right panel. 
plants inoculated with sap from one of the second-generation plants developed moderate photobleaching. $P D S$ silencing was maintained through one more round of mechanical inoculation but was lost after a third round. Remarkably, RT-PCR and sequencing of the insert remaining in the virus-infected plants showing PDS silencing after two rounds of sap-inoculation showed the same deletion as in the original second-generation plant (Fig. 4C).

\section{DISCUSSION}

In this report, we investigated the nature of transient silencing in barley using BSMV. After determining the optimal conditions for VIGS in barley, we investigated the impact of inserting different lengths of PDS in BSMV. Plants inoculated with BSMV carrying a $P D S$ fragment between 584 and 128 nt in length (Fig. 1B) gave rise to a 70 to $84 \%$ reduction in PDS mRNA compared with plants inoculated with $\mathrm{BSMV}_{\mathrm{WT}}$ (Fig. 2B). No significant differences in $P D S$ silencing were observed between the insert lengths tested, although plants inoculated with $\mathrm{BSMV}_{\mathrm{PDS} 128}$ did not display the completely white photobleached areas produced by the longer inserts (Fig. 2A and B). Equal silencing efficiencies with $P D S$ inserts varying between 1,215 and $185 \mathrm{nt}$ also were observed by Holzberg and associates (2002), and Scofield and associates (2005) observed consistent photobleaching in barley and wheat, with BSMV carrying $P D S$ fragments down to $120 \mathrm{nt}$, supporting the conclusion that, within the limits tested here, silencing efficiency is not strongly affected by insert length. Sense and antisense versions of the 400- and 128-nt fragments also produced similar levels of silencing, in agreement with previous studies (Holzberg et al. 2002; Lacomme et al. 2003).

In plants inoculated with $\mathrm{BSMV}_{\mathrm{WT}}$, we found an increase in $P D S$ mRNA levels compared with uninoculated control plants. This induction was consistently observed as a response to BSMV infection (Fig 2B; data not shown); hence, in plants inoculated with BSMV carrying fragments of $P D S$, an induction of $P D S$ must occur simultaneously with $P D S$ silencing. Consequently, all estimates of the downregulation of PDS mRNA in plants infected with BSMV-PDS constructs were calculated relative to plants infected with $\mathrm{BSMV}_{\mathrm{WT}}$. Induction of $P D S$ by BSMV has not been reported previously. Carotenoid biosynthesis pathway components, including $P D S$, have been shown to be induced in response to reactive oxygen species (ROS) (Bouvier et al. 1998). Production of ROS is a well-known element of hypersensitive reactions induced by incompatible interactions between plants and pathogens, but production of ROS also has been observed during compatible plant-virus interactions involving Tobacco mosaic virus in N. tabacum (Allan et al. 2001) and Cauliflower mosaic virus in Arabidopsis thaliana (Love et al. 2005). We speculate that BSMV infection similarly may lead to production of ROS in barley, with subsequent induction of $P D S$. Another possible cause for the induction of $P D S$ by BSMV may be related to the mosaic chlorosis typi-

A

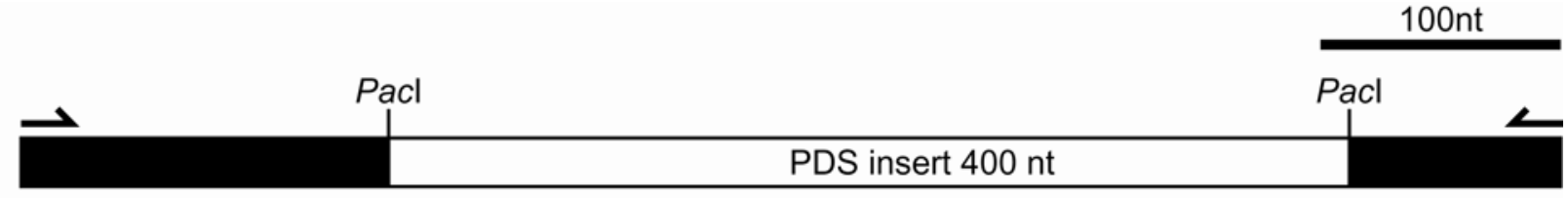

B

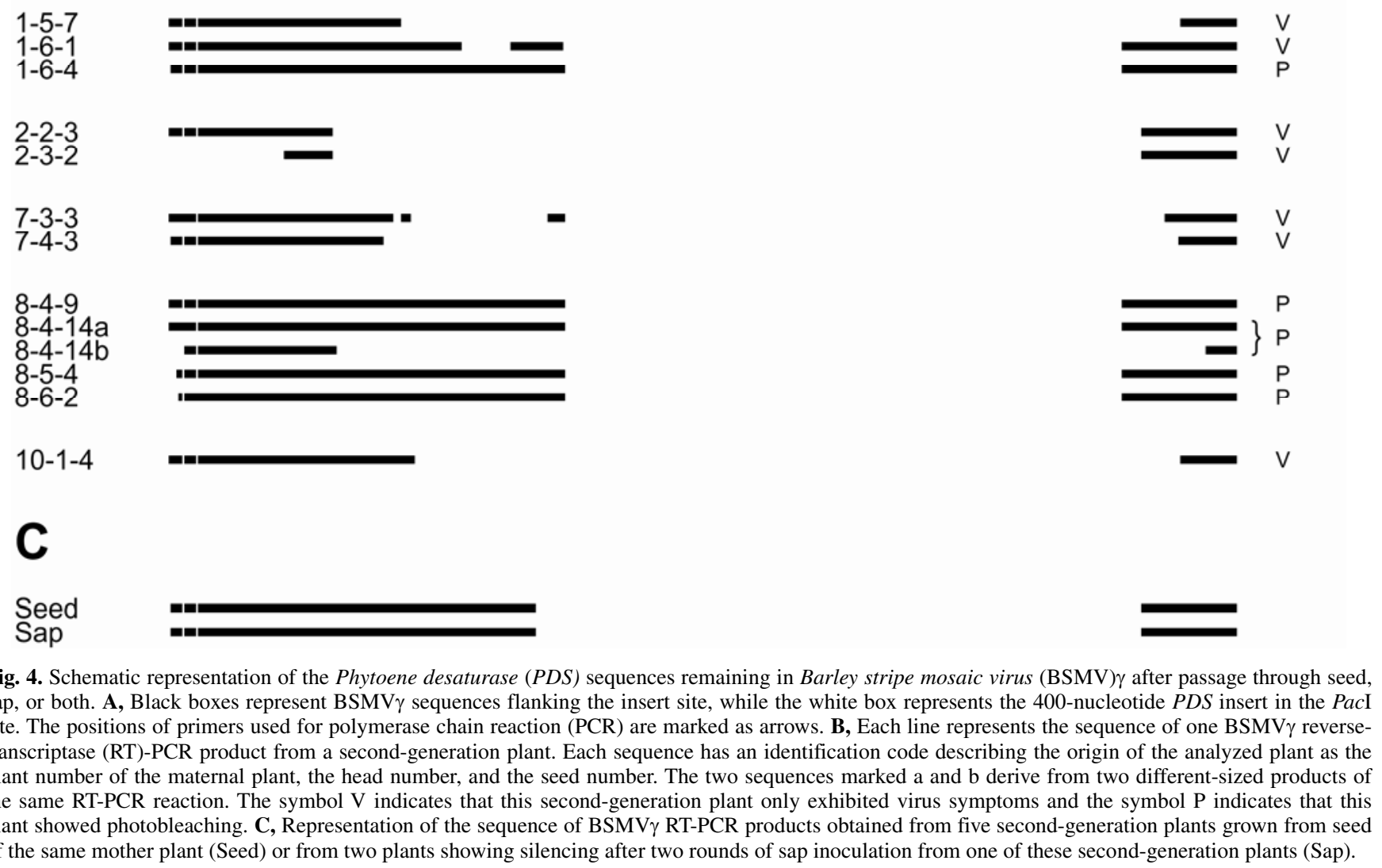


cally observed during BSMV infections in barley. Corona and associates (1996) observed that chemical inhibition of either carotenoid or chlorophyll biosynthesis led to induction of the $P D S$ promoter. These authors conclude that the $P D S$ promoter probably is regulated by end products in the carotenoid biosynthetic pathway, and that the carotenoid and chlorophyll biosynthesis pathways are interdependent. Thus, the BSMV-induced chlorosis may directly or indirectly upregulate transcription from the $P D S$ promoter. The end-product regulation of the $P D S$ promoter suggested by Corona and associates (1996) also implies that a further upregulation of $P D S$ mRNA transcription may take place in tissues undergoing virus-induced silencing of $P D S$, possibly leading to an underestimate of the VIGS efficiency.

Virus accumulation was significantly decreased in plants inoculated with $\mathrm{BSMV}_{\mathrm{PDS} 400}$ and $\mathrm{BSMV}_{\mathrm{PDS} 275}$ compared with plants inoculated with BSMV $\mathrm{WT}_{\mathrm{WT}}$ (Fig. 2C). On the other hand, accumulation of $\mathrm{BSMV}_{\mathrm{PDS} 584}$ or $\mathrm{BSMV}_{\mathrm{PDS} 128}$ was not significantly different from $\mathrm{BSMV}_{\mathrm{WT}}$. The virus concentration was determined 14 dpi. At this time, the PDS fragment in $\mathrm{BSMV}_{\mathrm{PDS} 584}$ was almost completely deleted, whereas most of the inserts carried by $\mathrm{BSMV}_{\mathrm{PDS} 400}, \mathrm{BSMV}_{\mathrm{PDS} 275}$, and $\mathrm{BSMV}_{\mathrm{PDS} 128}$ were retained (Fig. 2D). These data show that, for inserts above a certain length (approximately $130 \mathrm{nt}$ ), insert length is negatively correlated with virus accumulation. This generates a selection pressure leading to faster loss of the longest inserts. Large inserts also were lost more frequently than smaller inserts from PVX (Avesani et al. 2007). These results also suggest that shorter inserts are more likely to lead to longlasting silencing.

Some variation in the stability of the inserts was observed between experiments. For example, the time course experiments revealed early events of recombination of BSMV $\mathrm{PDS}_{400}$ deleting large parts of the insert by 5 dpi (Fig. 3D), whereas the same construct appeared relatively stable at $14 \mathrm{dpi}$ when used in the insert-length experiments (Fig. 2D). This variability may be related to growth conditions such as temperature. The time course experiments were conducted in a growth chamber with a constant temperature of $20^{\circ} \mathrm{C}$, whereas the insertlength experiments were conducted in a greenhouse with more variable temperature and lighting conditions. Temperature previously has been reported to influence the stability of inserts carried by recombinant viruses (Qu et al. 2005).

A significant reduction in PDS mRNA levels was observed 2 days earlier than the first visual photobleaching; thus, the onset of photobleaching was delayed compared with the reduction in PDS mRNA (Fig. 3A and B). This may be related to the turnover of the PDS protein; hence, the delay probably will vary with the half-life of the protein targeted by silencing. With the shortest $P D S$ fragment tested, typical photobleaching was not observed and the pale green or yellow phenotype developed later than the white photobleached areas induced by BSMV carrying larger $P D S$ fragments. Nevertheless, when measured at $14 \mathrm{dpi}, \mathrm{BSMV}_{\mathrm{PDS} 128}$ had reduced the $P D S$ mRNA level even more than BSMV carrying longer inserts. Our interpretation is that, in order to observe photobleaching, the PDS mRNA level must be lower than a certain threshold at a specific time point in plant development. If the threshold for silencing is met later than a critical time point in plant or leaf development, it will be possible to measure the reduction at the mRNA level but no visual photobleaching will be observed, perhaps because sufficient pools of carotenoids have accumulated. The threshold may be influenced by several factors, including the PDS expression level.

$P D S$ silencing was generally transient, with leaves developing after the onset of silencing showing less or no photobleaching. Furthermore, the photobleaching often was reversible, the green color being restored in previously photobleached areas.
However, passages of BSMV $\mathrm{PDS}_{400}$ through seed occasionally resulted in transfer of the silencing to the progeny plants. Likewise, VIGS could be transmitted mechanically through several passages. The loss of photobleaching with time was correlated with the gradual loss of the $P D S$ insert during the infection. The transient nature of $P D S$ silencing induced by BSMV in barley has been noted previously (Holzberg et al. 2002); however, the causes have not been investigated previously. Instability of virus vectors frequently is associated with the duplication of viral sequences such as subgenomic promoter sequences (Beck and Dawson 1990). In the BSMV vector used here, there are no duplicated sequences. However, long-distance interactions between terminal and internal elements in viral RNA genomes have been implicated in gene expression and replication of several plant viruses (Miller and White 2006), and the insertion of foreign sequences into the $3^{\prime}$ untranslated region of BSMV- $\gamma$ may interfere with such interactions. In the BSMV vector reported by Tai et al. (2005), the foreign sequences are inserted at the start of the $\gamma b$ gene. This may change the stability of the inserts compared with the vector used here; however, there is, at present, no data available on the stability of the inserts or of the silencing achieved using the vector of Tai and associates (2005). Furthermore, we have investigated only the stability of $P D S$ silencing; other target genes may show different kinetics of silencing. Indeed, recent studies suggest that the insert stability may be affected by the sequence of the insert (Zhong et al. 2005).

In plants inoculated with $\mathrm{BSMV}_{\mathrm{PDS128}}$, no areas with complete photobleaching were observed, although measurements of the PDS mRNA levels showed significant reductions with this construct (Fig. 2). Thus, in our experimental set-up, $128 \mathrm{nt}$ appeared to be close to the minimum insert length required for silencing. In second-generation plants showing silencing, RT-PCR analysis of the virus revealed $P D S$ fragments only up to 80 to $125 \mathrm{nt}$ (Fig. 4). We do not know if the critical silencing contribution came from the small pieces of insert left in the majority of the virus or if a subpopulation of the virus retaining a larger insert was the major donor of siRNA. Possibly, the contribution of siRNAs from the combined virus population must exceed a certain threshold in order to obtain silencing. In previous experiments with BSMV, consistent photobleaching was seen with inserts as short as $120 \mathrm{nt}$; however, reducing the insert down to $80 \mathrm{nt}$ resulted in photobleaching in only $16 \%$ of the barley plants (Scofield et al. 2005). However, short inserts of $80 \mathrm{nt}$ can be effective when present as a 40-nt inverted repeat (Lacomme et al. 2003). Related studies using PVX carrying part of GFP determined $23 \mathrm{nt}$ to be sufficient for silencing in a GFP transgenic $N$. benthamiana line, although longer inserts were more efficient (Thomas et al. 2001). In the same study, silencing of $P D S$ in $N$. benthamiana was obtained with PVX carrying PDS fragments down to $33 \mathrm{nt}$ in length (Thomas et al. 2001).

A detailed analysis of insert truncations after passaging of BSMV $_{\text {PDS400 }}$ through seed and sap revealed a nonrandom distribution, with independent deletion events often occurring at almost identical positions (Fig. 4). These apparent recombination hot spots might be related to primary or secondary structures in the virus or the insert (Nagy and Bujarski 1997; Olsthoorn et al. 2002).

In conclusion, our results demonstrate that the transient nature of VIGS using BSMV is dependent on the insert length. In several experiments, we observed reversion of $P D S$ silencing. This reversibility of the silencing was caused by major deletions in the insert in the $\gamma$ component of BSMV. Even after major deletions, silencing was observed as long as the remaining insert was approximately $100 \mathrm{nt}$. Furthermore, we demonstrated that, despite the instability of the BSMV vector, long-lasting silencing extending into second-generation plants is possible. This may 
open up possibilities of using the VIGS approach to study events related to flowering and seed filling in barley.

\section{MATERIALS AND METHODS}

The three full-length cDNA virus components from BSMV isolate ND18, each cloned behind a T7 promoter and designated $\operatorname{pBSMV}(\alpha-\mathrm{T} 7) \mathrm{ND} 18, \operatorname{pBSMV}(\beta-\mathrm{T} 7) \mathrm{ND} 18$, and $\operatorname{pBSMV}(\gamma-\mathrm{T} 7)$ ND18, were provided by A. O. Jackson (University of California, Berkeley, U.S.A.) (Petty et al. 1989). Restriction enzymes and enzymes used for modifications were handled according to the manufacturers instructions (Roche Diagnostics, Mannheim, Germany and New England Biolabs, Ipswich, MA, U.S.A.). Primers for RT-PCR and PCR were purchased at MWG Biotech AG (Ebersberg, Germany) and the virus construct sequences were verified using restriction analysis and sequencing at MWG.

\section{Introduction of a multiple cloning site in BSMV.}

We created the VIGS-competent BSMV multiple cloning site $\left(\mathrm{BSMV}_{\mathrm{MCS}}\right)$ by introducing a multiple cloning site in the $\gamma$ component immediately after the stop codon of the $\gamma \mathrm{b}$ gene by site-directed mutagenesis in an approach similar to that described by Holzberg and associates (2002). Through a series of subclonings, we introduced NheI before and SmaI, PacI, and BamHI after the $\gamma \mathrm{b}$ stop codon. The resulting sequence spanning the multiple cloning site of $\mathrm{BSMV}_{\mathrm{MCS}}$ was 5 -tcttccgttgcta gctaacccgggttaattaaggatccttagctagctaaaaa (the stop codon of the $\gamma \mathrm{b}$ gene is underlined). In this vector, fragments of genes for VIGS can be inserted using the unique restriction sites SmaI, PacI, or BamHI.

\section{Cloning of $P D S$ fragments.}

Barley RNA was isolated using the TriZOL method (Ambion, Austin, TX, U.S.A.), and DNase treated and used for RT-PCR using M-MLV reverse transcriptase (Invitrogen, Carlsbad, CA, U.S.A.) and Expand polymerase (Roche, Penzberg, Germany). Primers were designed using a partial $P D S$ sequence from barley, GenBank accession number AY062039 (Holzberg et al. 2002). Primers 5'-cccgggttaattaatctttgggtggtgaggtccggc and 5' ggatccttaattaagaattctctagacccttcgatcggcgaccgtt were used to amplify a 584-nt fragment, 5'-ggccttaattaactggataagttagtggga gtt and 5'-ggccttaattaaccettcgatcggcgaccgtt for the 400-nt fragment, 5'-ggatccttaattaagaattctctagaccettcgatcggcgaccgtt and 5'cccgggttaattaaacatgtctttagcgtgcaag for the 275-nt fragment, and primers 5 '-ccegggttaattaaggagtactatgatccaaaccgttcgatgttg gag and 5'-ggatccttaattaagaattctctagatcatctggaaacaacttggcta for the 128-nt fragment. The PCR products were TOPO-TA cloned into pCR 2.1-TOPO (Invitrogen) and verified by sequencing before insertion into the $\mathrm{PacI}$ site of $\mathrm{pBSMV}_{\mathrm{MCS}}$.

\section{BSMV inoculation in barley.}

The plasmids corresponding to the three components of BSMV were linearized with MluI ( $\alpha$ and $\gamma$ components) or SpeI ( $\beta$ component) and used as templates for in vitro transcription by the mMessage mMACHINE transcription kit (Ambion). The three viral RNA components were mixed in a 1:1:1 ratio and $3 \mu \mathrm{l}$ of combined transcript was mixed with $5 \mu \mathrm{l}$ of FES buffer (77 mM glycine, $60 \mathrm{mM} \mathrm{K} \mathrm{HPO}_{4}, 22 \mathrm{mM} \mathrm{Na}_{4} \mathrm{P}_{2} \mathrm{O}_{7} \cdot 10$ $\mathrm{H}_{2} \mathrm{O}$, and $1 \% \mathrm{wt} / \mathrm{vol}$ bentonite) and applied with gentle strokes to Carborundum-dusted leaves of barley 6 days postsowing. Unless stated otherwise, all barley plants were cv. Black Hulless grown in 11-cm pots with soil (Pindstrup Mix 2, Ryomgaard, Denmark), in either the greenhouse or growth chamber at $20^{\circ} \mathrm{C}$ with $16 \mathrm{~h}$ of light and $8 \mathrm{~h}$ of darkness. Greenhouse light intensities did not exceed 1,200 $\mu \mathrm{mol} \mathrm{m}^{-2} \mathrm{~s}^{-1}$ and were between 240 and $380 \mu \mathrm{mol} \mathrm{m}^{-2} \mathrm{~s}^{-1}$ in the growth chambers.

\section{Relative measurements \\ of PDS mRNA and BSMV using qRT-PCR.}

The complete laminas of plant leaves were harvested individually and frozen in liquid $\mathrm{N}_{2}$. The RNA was extracted with an RNeasy Plant Mini Kit (Qiagen, Germantown, MD, U.S.A.) with on-column DNase digestion (Qiagen). The RNA concentration was measured with a NanoDrop ND-1000 spectrophotometer (NanoDrop Technologies, Wilmington, DE, U.S.A.). cDNA was prepared with Expand reverse transcriptase (Roche) using RNA at $4 \mathrm{ng} / \mu \mathrm{l}$ for insert length experiments or $5 \mathrm{ng} / \mu \mathrm{l}$ for time course experiments and RNasin (Promega, Madison, WI, U.S.A.) at $4 \mathrm{U} / \mu \mathrm{l}$. The relative amounts of RNA were measured by qRT-PCR with primers and probes labeled with FAM (5') and blackhole quencher ( $\left.3^{\prime}\right)$. The primers and probes were specific for BSMV $\gamma$ (P3: forward, 5'-aactgccaatcgtgagta ggtt; reverse, 5'-ctcctgttcagaacgtttcagaagt; probe 5'-ctgtggccaa aagcat), PDS outside the region inserted in BSMV (P1: forward, 5'-ccatgatatttgccatgccaaacaa; reverse, 5'-ggtgccggcaaagt ctct; probe, $5^{\prime}$-atacagccgtttgatttc), and $P D S$ partly overlapping the 400-nt $P D S$ insert (P2: forward, 5'-tgtaccacaagagtggagaga gatc; reverse, $5^{\prime}$-tgatgacaggaactcccactaactt; probe, $5^{\prime}$-cagcctctt gaaataag). The $18 \mathrm{~S}$ rRNA (forward, 5'-ggaatgcctagtaagcgag tca; reverse, 5'-cgggcggtgtgtacaaag; probe, 5'-cagggacgtagtca acg) or $G A P D H$ (forward, 5'-ttgttgatctcactgttagaacc; reverse, $5^{\prime}$-ctgctgtcaccaacgaag; probe, $5^{\prime}$-acatcaagaaggctatcaaggctgcttcc) was used for normalization. All primers and probes for qRTPCR were purchased from Applied Biosystems (Foster City, CA, U.S.A.) or MWG and validated to have PCR efficiencies between 91 and 98\%, as recommended by Applied Biosystems, except the primer/probe set for quantification of BSMV (P3) (see below). P3 was placed $158 \mathrm{nt}$ upstream of the multiple cloning site in $\gamma b$ to obtain a quantitative measurement of the VIGS-inducing component of BSMV. This primer/probe set was validated to have a nonlinear PCR efficiency of $89 \%$ when using undiluted RT samples. As a result, all real-time analyses using the BSMV primer/probe set were performed using a $1 / 10$ dilution of the RT mix, resulting in a PCR efficiency close to $100 \%$. Relative quantification of the cDNA and validations of primer/probe sets were performed on an $\mathrm{ABI}$ 7500 (Applied Biosystems) using Taqman universal PCR master mix (Applied Biosystems) according to the manufacturer's recommendations. All samples were measured in duplicate (insert length experiments) or triplicate (time course experiments) and the resulting data were normalized to $18 \mathrm{~S}$ rRNA using the $\Delta \Delta C T$ method (Livak and Schmittgen 2001).

\section{Insert stability.}

PCR analysis and sequencing were performed to determine the stability of the PDS inserts in BSMV $\gamma$. The cDNA prepared for qRT-PCR was amplified using Expand polymerase (Roche) and a pair of primers flanking the MCS in BSMV $\gamma$ : 5 '-gaagaagatgcaggagctgaa and 5'-cactcccatcatatggttgat (Fig. 4). The resulting PCR products were excised from agarose gels, purified with the GFX PCR DNA and gel band purification kit (Amersham, Freiberg, Germany), and sequenced at MWG. The sequences were aligned using the local multiple alignment program Muscle run on a local computer (Edgar 2004).

\section{ACKNOWLEDGMENTS}

We gratefully acknowledge A. O. Jackson (University of California, Berkeley, U.S.A.) for providing the BSMV cDNA clones. Our colleagues M. H. Dinesen, J. A. B. Strickertsson, B. S. Olsen, M. L. Stephensen, and D. B. Collinge are highly appreciated for assistance and for valuable discussions. This work was financed by The Danish Veterinary and Agricultural Research Council (SJVF), The Danish Research School for Biotechnology (FOBI), and the Danish National Research Foundation. 


\section{LITERATURE CITED}

Agranovsky, A. A., Dolja, V. V., Kagramanova, V. K., and Atabekov, J. G. 1979. Presence of a cap structure at the $5^{\prime}$-end of barley stripe mosaicvirus RNA. Virology 95:208-210.

Agranovsky, A. A., Dolja, V., V., and Atabekov, J. G. 1982. Structure of the 3' extremity of barley stripe mosaic-virus RNA-evidence for internal poly(a) and a 3'-terminal transfer RNA-like structure. Virology 119:51-58.

Allan, A. C., Lapidot, M., Culver, J. N., and Fluhr, R. 2001. An early Tobacco mosaic virus-induced oxidative burst in tobacco indicates extracellular perception of the virus coat protein. Plant Physiol. 126:97-108.

Altpeter, F., Vasil, V., Srivastava, V., Stoger, E., and Vasil, I. K. 1996. Accelerated production of transgenic wheat (Triticum aestivum L) plants. Plant Cell Rep. 16:12-17.

Avesani, L., Marconi, G., Morandini, F., Albertini, E., Bruschetta, M., Bortesi, L., Pezzotti, M., and Porceddu, A. 2007. Stability of Potato virus $X$ expression vectors is related to insert size: Implications for replication models and risk assessment. Transgenic Res. 16:587-597.

Beck, D. L., and Dawson, W. O. 1990. Deletion of repeated sequences from tobacco mosaic virus mutants with two coat protein genes. Virology 177:462-469.

Bouvier, F., Backhaus, R. A., and Camara, B. 1998. Induction and control of chromoplast-specific carotenoid genes by oxidative stress. J. Biol. Chem. 273:30651-30659.

Bragg, J. N., and Jackson, A. O. 2004. The C-terminal region of the Barley stripe mosaic virus gammab protein participates in homologous interactions and is required for suppression of RNA silencing. Mol. Plant Pathol. 5:463-481.

Brigneti, G., Martin-Hernandez, A. M., Jin, H., Chen, J., Baulcombe, D. C., Baker, B., and Jones, J. D. 2004. Virus-induced gene silencing in Solanum species. Plant J. 39:264-272.

Brodersen, P., and Voinnet, O. 2006. The diversity of RNA silencing pathways in plants. Trends Genet. 22:268-280.

Burch-Smith, T. M., Anderson, J. C., Martin, G. B., and Dinesh-Kumar, S. P. 2004. Applications and advantages of virus-induced gene silencing for gene function studies in plants. Plant J. 39:734-746.

Constantin, G. D., Krath, B. N., MacFarlane, S. A., Nicolaisen, M., Johansen, I. E., and Lund, O. S. 2004. Virus-induced gene silencing as a tool for functional genomics in a legume species. Plant J. 40:622-631.

Corona, V., Aracri, B., Kosturkova, G., Bartley, G. E., Pitto, L., Giorgetti, L., Scolnik, P. A., and Giuliano, G. 1996. Regulation of a carotenoid biosynthesis gene promoter during plant development. Plant J. 9:505-512.

Dalmay, T., Hamilton, A., Rudd, S., Angell, S., and Baulcombe, D. C. 2000. An RNA-dependent RNA polymerase gene in Arabidopsis is required for posttranscriptional gene silencing mediated by a transgene but not by a virus. Cell 101:543-553.

Ding, X. S., Schneider, W. L., Chaluvadi, S. R., Mian, M. A., and Nelson, R. S. 2006. Characterization of a Brome mosaic virus strain and its use as a vector for gene silencing in monocotyledonous hosts. Mol. PlantMicrobe Interact. 19:1229-1239.

Edgar, R. C. 2004. MUSCLE: Multiple sequence alignment with high accuracy and high throughput. Nucleic Acids Res. 32:1792-1797.

Edwards, M. C. 1995. Mapping of the seed transmission determinants of barley stripe mosaic virus. Mol. Plant-Microbe Interact. 8:906-915.

Gustafson, G., and Armour, S. L. 1986. The complete nucleotide sequence of RNA beta from the type strain of barley stripe mosaic virus. Nucleic Acids Res. 14:3895-3909.

Hein, I., Barciszewska-Pacak, M., Hrubikova, K., Williamson, S., Dinesen, M., Soenderby, I. E., Sundar, S., Jarmolowski, A., Shirasu, K., and Lacomme, C. 2005. Virus-induced gene silencing-based functional characterization of genes associated with powdery mildew resistance in barley. Plant Physiol. 138:2155-2164.

Holzberg, S., Brosio, P., Gross, C., and Pogue, G. P. 2002. Barley stripe mosaic virus-induced gene silencing in a monocot plant. Plant $\mathrm{J}$. 30:315-327.

Kumagai, M. H., Donson, J., della-Cioppa, G., Harvey, D., Hanley, K., and Grill, L. K. 1995. Cytoplasmic inhibition of carotenoid biosynthesis with virus-derived RNA. Proc. Natl. Acad. Sci. U.S.A. 92:1679-1683.

Lacomme, C., Hrubikova, K., and Hein, I. 2003. Enhancement of virusinduced gene silencing through viral-based production of invertedrepeats. Plant J. 34:543-553.

Landry, P., and Perreault, J. P. 2005. Identification of a peach latent mosaic viroid hairpin able to act as a Dicer-like substrate. J. Virol. 79:6540-6543.

Liu, Y., Schiff, M., and Dinesh-Kumar, S. P. 2002. Virus-induced gene silencing in tomato. Plant J. 31:777-786.

Livak, K. J., and Schmittgen, T. D. 2001. Analysis of relative gene expression data using real-time quantitative PCR and the 2(-delta delta $\mathrm{C}(\mathrm{T})$ ) method. Methods 25:402-408.
Love, A. J., Yun, B. W., Laval, V., Loake, G. J., and Milner, J. J. 2005. Cauliflower mosaic virus, a compatible pathogen of Arabidopsis, engages three distinct defense-signaling pathways and activates rapid systemic generation of reactive oxygen species. Plant Physiol. 139:935-948.

Miller, W. A., and White, K. A. 2006. Long-distance RNA-RNA interactions in plant virus gene expression and replication. Annu. Rev. Phytopathol. 44:447-467.

Molnar, A., Csorba, T., Lakatos, L., Varallyay, E., Lacomme, C., and Burgyan, J. 2005. Plant virus-derived small interfering RNAs originate predominantly from highly structured single-stranded viral RNAs. J. Virol. 79:7812-7818.

Nagy, P. D., and Bujarski, J. J. 1997. Engineering of homologous recombination hotspots with AU-rich sequences in brome mosaic virus. J. Virol. 71:3799-3810.

Olsthoorn, R. C., Bruyere, A., Dzianott, A., and Bujarski, J. J. 2002. RNA recombination in Brome mosaic virus: Effects of strand-specific stemloop inserts. J. Virol. 76:12654-12662.

Petty, I. T., and Jackson, A. O. 1990. Mutational analysis of barley stripe mosaic virus RNA beta. Virology 179:712-718.

Petty, I. T., Hunter, B. G., Wei, N., and Jackson, A. O. 1989. Infectious barley stripe mosaic virus RNA transcribed in vitro from full-length genomic cDNA clones. Virology 171:342-349.

Petty, I. T., French, R., Jones, R. W., and Jackson, A. O. 1990. Identification of barley stripe mosaic virus genes involved in viral RNA replication and systemic movement. EMBO (Eur. Mol. Biol. Organ.) J. 9:3453-3457.

Qu, F., Ye, X., Hou, G., Sato, S., Clemente, T. E., and Morris, T. J. 2005. RDR6 has a broad-spectrum but temperature-dependent antiviral defense role in Nicotiana benthamiana. J. Virol. 79:15209-15217.

Ratcliff, F., Martin-Hernandez, A. M., and Baulcombe, D. C. 2001. Technical advance. Tobacco rattle virus as a vector for analysis of gene function by silencing. Plant J. 25:237-245.

Ruiz, M. T., Voinnet, O., and Baulcombe, D. C. 1998. Initiation and maintenance of virus-induced gene silencing. Plant Cell 10:937-946.

Schwach, F., Vaistij, F. E., Jones, L., and Baulcombe, D. C. 2005. An RNA-dependent RNA polymerase prevents meristem invasion by $\mathrm{Po}$ tato virus $X$ and is required for the activity but not the production of a systemic silencing signal. Plant Physiol. 138:1842-1852.

Schweizer, P., Christoffel, A., and Dudler, R. 1999a. Transient expression of members of the germin-like gene family in epidermal cells of wheat confers disease resistance. Plant J. 20:541-552.

Schweizer, P., Pokorny, J., Abderhalden, O., and Dudler, R. 1999b. A transient assay system for the functional assessment of defense-related genes in wheat. Mol. Plant-Microbe Interact. 12:647-654.

Schweizer, P., Pokorny, J., Schulze-Lefert, P., and Dudler, R. 2000. Technical advance. Double-stranded RNA interferes with gene function at the single-cell level in cereals. Plant J. 24:895-903.

Scofield, S. R., Huang, L., Brandt, A. S., and Gill, B. S. 2005. Development of a virus-induced gene-silencing system for hexaploid wheat and its use in functional analysis of the Lr21-mediated leaf rust resistance pathway. Plant Physiol. 138:2165-2173.

Shen, Q. H., Saijo, Y., Mauch, S., Biskup, C., Bieri, S., Keller, B., Seki, H., Ulker, B., Somssich, I. E., and Schulze-Lefert, P. 2007. Nuclear activity of MLA immune receptors links isolate-specific and basal disease-resistance responses. Science 315:1098-1103.

Tai, Y. S., Bragg, J., and Edwards, M. C. 2005. Virus vector for gene silencing in wheat. Biotechniques 39:310, 312, 314.

Tai, Y.-S., Bragg, J., and Meinhardt, S. W. 2007. Functional characterization of ToxA and molecular identification of its intracellular targeting protein in wheat. Am. J. Physiol. 2:76-89.

Thomas, C. L., Jones, L., Baulcombe, D. C., and Maule, A. J. 2001. Size constraints for targeting post-transcriptional gene silencing and for RNA-directed methylation in Nicotiana benthamiana using a Potato virus $X$ vector. Plant J. 25:417-425.

Timian, R. G. 1974. Range of symbiosis of barley and barley stripe mosaic virus. Phytopathology 64:342-345.

Voinnet, O., Lederer, C., and Baulcombe, D. C. 2000. A viral movement protein prevents spread of the gene silencing signal in Nicotiana benthamiana. Cell 103:157-167.

Zhong, X., Hou, H., and Qiu, W. 2005. Integrity of nonviral fragments in recombinant Tomato bushy stunt virus and defective interfering RNA is influenced by silencing and the type of inserts. Mol. Plant-Microbe Interact. 18:800-807.

Zhou, H., and Jackson, A. O. 1996. Expression of the barley stripe mosaic virus RNA beta "triple gene block." Virology 216:367-379.

Zuker, M., Mathews, D. H., and Turner, D. H. 1999. Algorithms and Thermodynamics for RNA Secondary Structure Prediction: A Practical Guide in RNA Biochemistry and Biotechnology. Kluwer Academic Publishers, Dordrecht, The Netherlands. 